\title{
High-throughput genotyping and transcriptome analysis reveals candidate genes associated with wheat heading time
}

Kiseleva A.A.*, Muterko A.F., Salina E.A.

Institute of Cytology and Genetics, SB RAS, Novosibirsk, Russia

*e-mail: antkiseleva@bionet.nsc.ru

Variability of heading time may affect wheat adaptation to different environments. Thus, the detection of new heading time determinants is important for improving cereals. In this work, we used common wheat cultivar Chinese Spring (CS) and the substitution line of CS with 5B chromosome from $T$. dicoccoides (CS-5Bdic), different in their flowering time by two weeks, to detect determinants of heading time on $5 \mathrm{~B}$ chromosome. Using the RICL population from a cross of CS x CS-5Bdic, we detected QTL in pericentromeric region of chromosome $5 \mathrm{~B}$, that was significantly associated with heading time. To determine candidate genes, that affect the trait of our interest, we analyzed transcriptomes of CS and CS-5Bdic using the RNA-seq. Three replicate samples from each genotype were harvested at four time points over 24 hours since the beginning of the light period $(0,3,9$ and 16 hours). The genes showed differential expression between the substituted line and CS were identified in each time point. GO analysis revealed that the DEGs were mainly involved in nitrogen assimilation and metabolism, photosynthesis, regulation of transcription, ATP metabolism. Among the genes, differentially expressed between CS and CS-5Bdic, one of the most interesting is TraesCS5B01G075300, which is higher expressed in CS at all time-points. This gene is localized in the region of the heading time QTL, detected previously. qPCR confirmed the revealed differences in the expression level. The TraesCS5B01G075300 gene encodes Myb transcription factor. Best hit resulted from blastp analysis against $A$. thaliana is EFM (EARLY FLOWERING MYB PROTEIN) protein, involved in regulation of flowering.

Acknowledgements: The study has been supported by the Budget project 0324-20190039 . 